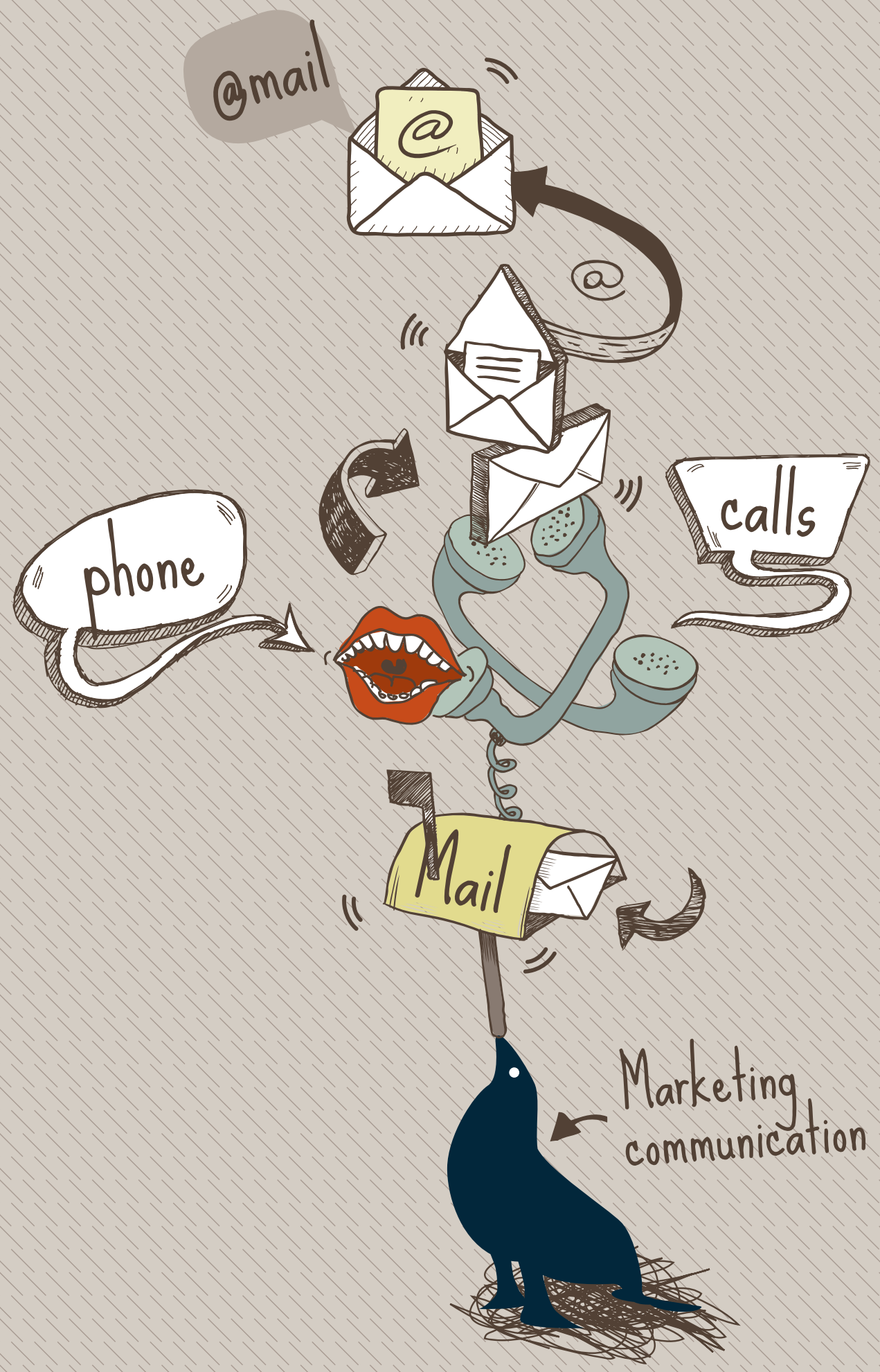

XX Well balanced communication channels improve repurchase rates. 


\title{
When Is Enough Enough? BALANCING ON THE FINE LINE IN MULTICHANNEL MARKETING COMMUNICATIONS
}

\author{
Andrea Godfrey Flynn, Kathleen Seiders and Glenn B. Voss
}

In an effort to build long-term, profitable relationships, many companies systematically engage in multichannel relational communication - personalized messages sent to existing customers through various channels as part of a broader relationship marketing strategy. However, too much of a good thing might actually ultimately have a bad effect. Whether ongoing direct communication with customers is perceived positively depends on its volume, the mix of communication channels, and the alignment of those channels with customers' preferences. There is an ideal level of communication. If it is exceeded, customers react negatively and this negative response can be exacerbated by the use of multiple channels. The ideal level differs depending on individual channel preferences. Aligning channels with customer preferences is advisable to optimize repurchase rates.

\section{Not This Again!}

When companies aim to build relationships with customers, they contact them through various channels - email, phone, direct mail, and so on. Initially, personalized communications lead consumers to reciprocate these efforts, and at a certain point, communication is ideal in its amount and appeal. But past that point, consumers start to reject more communications from a company, which is exactly the opposite reaction to what the company wants. To avoid such a negative response, marketers need to align their communication channels appropriately with the preferences consumers express to ensure that they are doing just enough and no more.

For marketers, especially those that aim to develop some form of long-term relationship with their customers, finding the best means for moving from solicitation to sale is a constant challenge. Does customer $\mathrm{X}$ prefer a personalized phone call before she will repurchase? How often does customer $Y$ want to receive email reminders? And is it worthwhile to send out mailed solicitations to both of them, so they have something physical to remind them to buy?

\section{In Search of the Ideal Point of Communication}

For many companies, all of these questions are pertinent, because they engage in multichannel relational communications. Their goal is to address individual customers personally through a variety of channels, which ideally encourages those customers to stay in a relationship with the company and perhaps even expand their purchases. Depending on the customer, the communications might announce a new store opening, trumpet a new innovation, suggest a necessary service visit, offer a promotional discount, or even ask about satisfaction
THE AUTHORS

Andrea Godfrey Flynn, Associate Professor of Marketing, University of San Diego, San Diego, CA, USA andreaflynn@sandiego.edu

Kathleen Seiders, Professor of Marketing, Boston College, Boston, MA, USA

kathleen.seiders@bc.edu

Glenn B. Voss, Marilyn and Leo Corrigan Endowed Professor of Marketing, Southern Methodist University, Dallas, TX, USA. gvoss@smu.edu

The article is an adapted version of Godfrey, Andrea; Seiders, Kathleen; Voss, Glenn B. (2011): "Enough is Enough! The Fine Line in Executing Multichannel Relational Communication", Journal of Marketing, Vol. 75 (4), pp. $94-109$, and is published with the permission of the American Marketing Association. 
$\{$ Box 1 $\}$

\section{A QUICK EXAMPLE}

Politicians running for office in many democratic countries spend millions on their campaigns. In the 2012 U.S. presidential campaign for example, total spending by all candidates - in the primaries as well as the ultimate two-party race - is expected to move beyond the US $\$ 8$ billion mark. The spending spreads across various communication media. Television commercials are targeted to different geographic areas. In key battleground states, candidates' advertisements can take up as much as half the advertising airtime in the weeks leading up to a primary election.

Each candidate also maintains a website that sends frequent email messages to people who register. Thus an interested voter who registers with a single national party but also visits the websites of several candidates for that party's nomination could receive multiple, competing messages all at the same time. Telephone contacts also are common; in recent years, "robo-calls" have earned special scorn from call recipients, who pick up the phone only to hear a recorded message.

Direct mailing campaigns are diminishing in general as a percentage of total advertising spending, but pamphlets and solicitations still appear in voters' mailboxes. This vast provision of information leads many voters to proclaim, "That's enough! I'm sick of all the candidates, and the election is still months away".

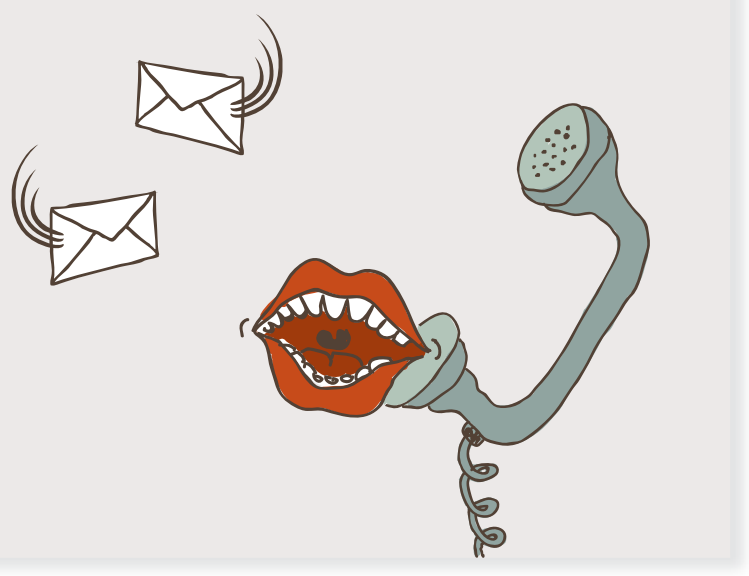

after an encounter. All of these contacts seem promising in theory, but in reality, many customers express irritation, frustration and annoyance with repeated contacts that make it seem as if the company is refusing to leave them alone.

A key question is whether the effects of communications across various channels add up (i.e., mail + phone + email) or are multiplied in consumers' minds. Does communication through multiple channels increase responses from consumers, because the company shows how dedicated it is to the relationship, or does it alienate them by seeming impersonal and intrusive? Our study (Box 3) indicates that the answer is both. That is, up to a certain volume of communication, consumers appreciate various forms of communication. By issuing contacts through various channels, the company demonstrates its dedication to the customer relationship. It can reach an ideal point for customer retention and satisfaction by providing a level of communication that encourages the most purchase behavior without overloading consumers. This ideal point is different for various channels, consumers, and their combinations. Then past this point, more communication leads to customer reactance - that is, the customer says "enough!" and begins to reject both the communication and the relationship with the company.

For marketers, the general consensus is that some communication is better than none. But what is the ideal amount? To find this level, the marketer needs to determine the impact of specific communication channels (e.g., email, person-to-person, telephone, direct mail), both individually and in combination. Their focus should not be simply the aggregate volume of contacts, because this view prevents them from finding the best means to manage the mix of communication channels. In addition, companies should develop protocols and guidelines for limiting total communication to customers. Finally, they can improve their effectiveness by defining the best channel combinations.

\section{Setting the Right Volume}

Let's start with the question of the best volume of communication, before considering how to mix the channels and also align them with consumers' preferences. The relationship between levels of communication and customer satisfaction follows an inverted 
$\{$ Box 2$\}$

\section{EXPERIENCES OF A MAJOR GIFTS OFFICER WITH A NATIONAL NON-PROFIT AGENCY}

"I spent two months assessing and segmenting the database of past donors and creating a plan for scheduling personal meetings with 60 high-gift-capacity donors. Ten donors were leery about speaking with me. I left messages for another 30 donors, but only three returned my call. Five donors hung up on me with varying degrees of anger. One person said that I was the third person to call in three days. Thirteen donors also received a handwritten note to inform them of our fundraising campaign. In the end, I did not book a single appointment. Because our organization relies heavily on phone and mail contacts along with face-to-face fundraising, potential donors have already been bombarded by mail and phone calls by the time I try to arrange a personal meeting. I am removing all of my donors from the phone and mail contact lists to control how much communication they receive from us."

$\{$ Box 3 $\}$

\section{COMBINING SURVEY DATA WITH CUSTOMER CONTACT HISTORY AND REPURCHASE BEHAVIOR}

The survey sampling frame included 3,370 randomly selected customers of a large automobile dealership in the USA with a high volume service department who had visited the service department within the past year. Of the invited customers, $36 \%$ returned their questionnaire on their channel preferences. This data was matched with 39 months of customer contact history and repurchase behaviour allowing for a longitudinal analysis. The combined data was used to estimate a system of simultaneous equations in a maximum likelihood analysis. Repurchase visits and purchase spending served as dependent variables to capture customer response to relational communication. Independent variables included the self-report measure of channel preference and the number of contacts to each customer per phone, email or regular mail from the company contact data. 


\section{FIGURE 1:}

Ideal Points for the Impact of Relational Communication on Repurchase Spending

\section{PANEL A}

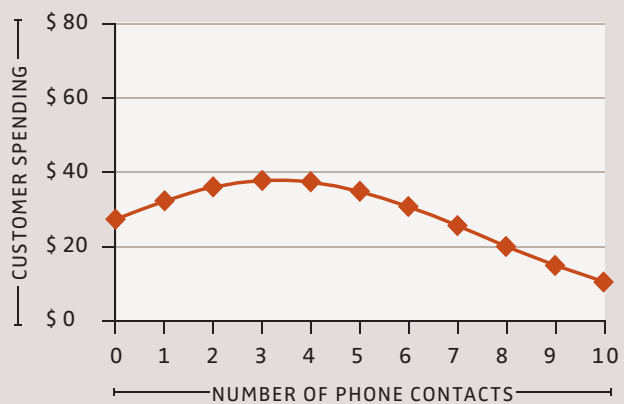

PANEL B

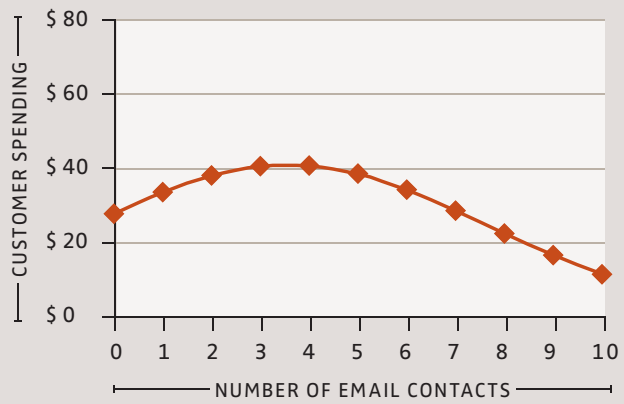

PANEL C

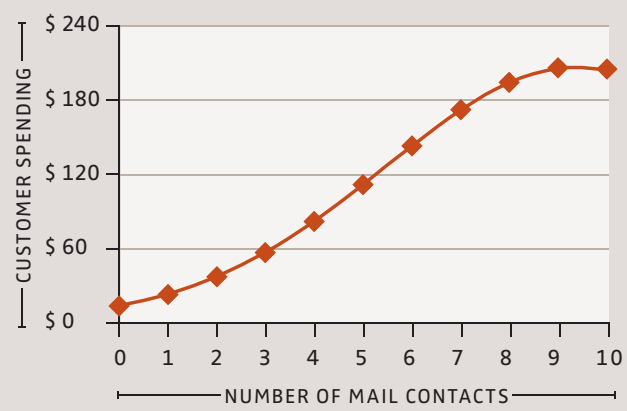

Repurchase spending captures average customer expenditures on automotive maintenance and repair services over a 3-month period
The data from a three-year study of a car dealership's automotive service business support this general notion. For three types of communication channels (phone, email, and regular mail), relational contacts have positive impacts on repurchase rates, up to an ideal point. Figure 1 shows these separate curves, all of which are inverted U-shaped. The first few initial contacts prompt positive responses, and customers buy more. But after approximately three phone calls, four emails, and ten direct mailings over a three month period, customers start to exhibit some reactance. (Figure 1 , next page)

Reactance occurs when an influence attempt causes the opposite reaction in the target. Such effects are common in interpersonal relationships, but they also arise in consumer settings. When consumers perceive that heavyhanded marketing efforts are attempting to manipulate or limit their freedom of choice, they refuse to act in the way marketing encourages them to. In extreme cases, they may even exhibit a boomerang or backlash effect. For example, in democratic societies, many voters become disenchanted with political advertising that they consider manipulative and therefore refuse to participate in electoral processes at all.

A similar effect appears to arise in the automotive service setting. When the dealership worked "too hard" to contact them through more than three telephone calls, four emails, or ten direct mail messages, customers stopped repurchasing. That is, they reacted negatively to an excessive volume of marketing communication.

\section{Setting the Right Mix}

Of course, few marketers would limit themselves to a single communication channel, unless they were forced to do so. The plethora of possibilities for reaching consumers makes multichannel relational marketing a norm in many industries. Therefore, the next step is to determine how the various channels interact and work together to exert an influence on customers' reactions to marketing communications. That is, if a customer receives a phone call, an email and a brochure in the mail all in the same week, will they be pleased that the seller is displaying serious interest, or be annoyed by the blitzkrieg of information? 
The contact, survey, and repurchase data reveal that in most cases, the interaction between various communication channels have notable effects. In particular, they move the position of the ideal point of communication volume. When the dealership combines telephone with email messages, the repurchase rate declines. If it issues both email and regular mail, it can suffer a similar decline in repurchasing among its consumers. Although the interaction of phone and direct mail contacts has a negative effect too, the impact was not significant for the data in this study.

In addition, the amount of total communication that the customer will accept before exhibiting reactance also decreases. For the phone $x$ email combination, the ideal point moves down. For example, if the dealership makes one telephone contact, the ideal number of email contacts is five to six. However, if it adds a few more telephone calls to its mix, the ideal number of email declines to two to three. Similarly, when there is one mail contact, the ideal number of email contacts is approximately five, but with five mail contacts, the ideal number of email contacts drops down to one.

\section{Setting Levels in Accordance with Customer Preferences}

The final set of insights, derived from the combined data from customers, the dealership and sales records, indicate that marketers need to take careful consideration of the preferences that consumers express about communications as well. Quite simply, preference for a channel improves customers' responses to communications through that channel. For example, among customers who prefer email contacts, repurchase rates are highest when they receive five email messages. Among consumers who do not prefer that channel, the ideal point that drives the highest repurchase rates is between two and three email contacts.

\section{Summary of Results}

This research offers three key findings for marketers:

1. There is an ideal volume of relational marketing communication, though that level varies across different communication channels. Before the ideal point, consumers increasingly reciprocate greater marketing communications. After the ideal point, they begin to exhibit reactance and show increasingly negative responses.

2. The interactions of the various channels have negative effects. That is, the ideal level for one type of communication drops when communication volume in the other channels increases. The multiplicative effects are stronger in some combinations than in others, but they are consistently negative.

3. Customers' preferences for certain channels affect the ideal rate of communication volumes on repurchase.

Such findings seem unlikely to apply in some other channels though. For example, if a television viewer can leave the room or change the channel rather than watch yet another commercial, then reactance may be less likely. These results suggest that control over the receipt of the message is a critical component of customers' responses to marketing communications. Reactance appeared most quickly in response to the telephone channel, less so in the email channel, and slowest in the regular mail channel.
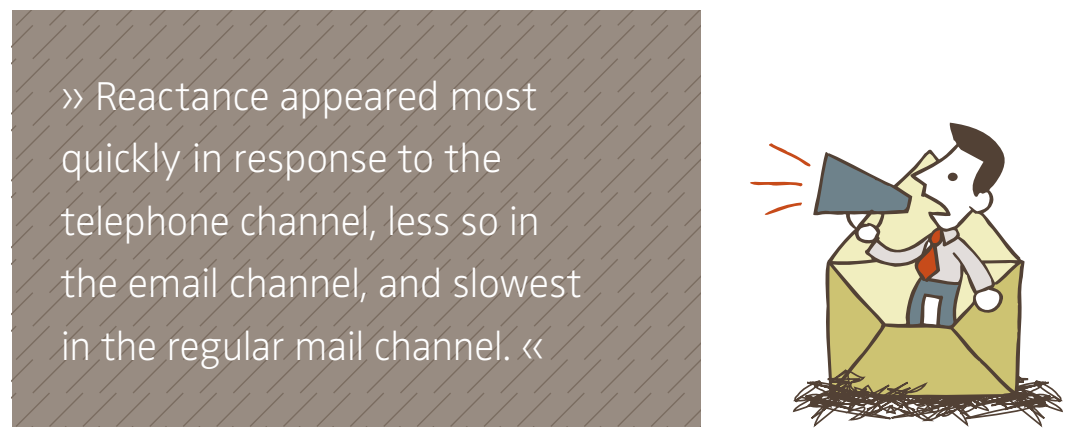
FIGURE 2:

Simulated Results of Three Relational Communication Strategies over a 3-Month Period

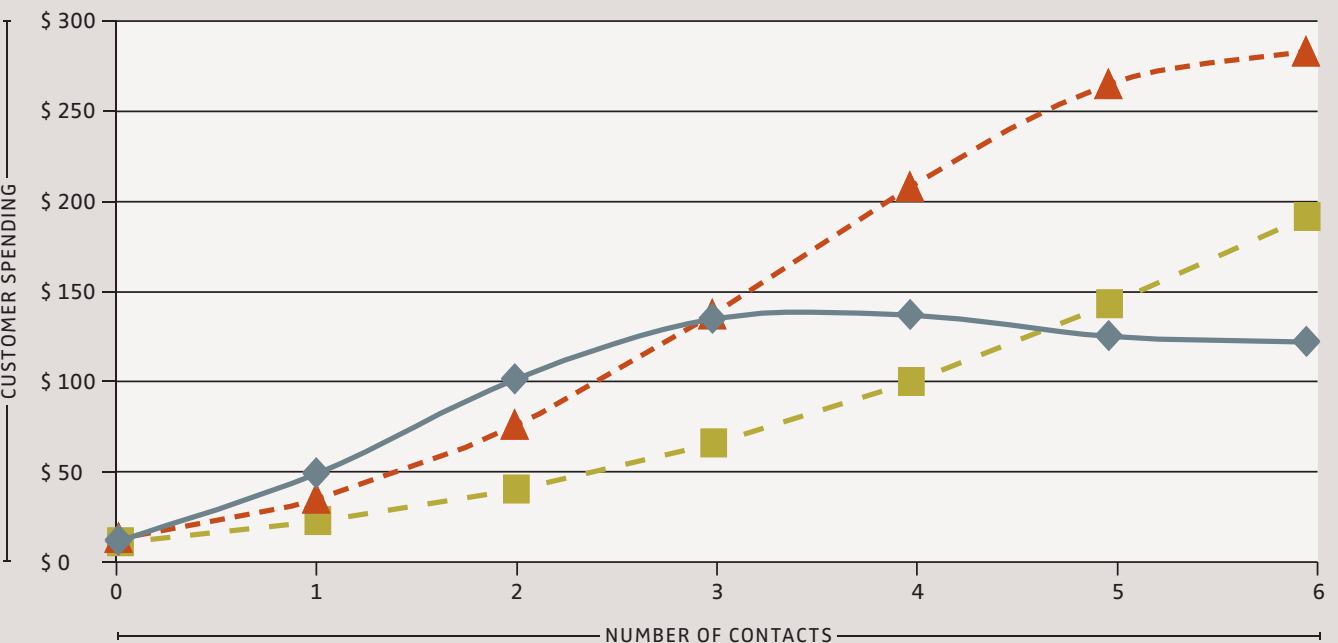

$-\mathcal{-}-$ Phone $=$ Mail, No email $\quad-\quad-$ Email, no phone or mail one...

two...

three...

Perhaps these findings reflect the level of perceived intrusiveness associated with each marketing communication channel. That is, a telephone call seems highly intrusive: it disrupts the recipient's immediate activities in real time. Even if the person ignores the call, the resulting voicemail message requires some action, even if just to dial into a voicemail system to delete the unwanted message. Communication managers should therefore limit all telephone contacts; for the company in this research setting, telephone contacts were most effective when limited to three contacts per quarter, on average.

Email appears rather less intrusive. It does not require a real-time disruption, and it is relatively easy to delete. In addition, customers have a simple and effective means for preventing messages in the future by flagging the sender and directing future communications to a junk mail folder. Thus, the car dealership's email contacts only prompted reactance if they exceeded four per quarter. Finally, postal mail seems least intrusive. It is easy to ignore and dispose of, and marketers can therefore safely send a higher number of mailings per quarter before they run the risk of customer reactance.

\section{Managerial Implications}

$>$ Monitor contact volume and channel combinations Marketing communication cannot succeed if it only seeks to avoid negative reactance; it also needs to provoke positive reciprocity. To maximize repurchase and minimize the likelihood of reactance, managers need to monitor their total contact volume with customers and also explore how their specific channel combinations alter the ideal point. Email combines poorly with telephone and mail contacts. However, customers respond relatively well to a combination of telephone and mail contacts - though this combination may be more expensive than those that rely more heavily on email.

\section{$>$ Adapt communication channels to preferences} Whenever possible, marketers also need to determine just which communication channel their customers prefer. The data in this study implied two main customer segments: the traditionalists, who respond positively to traditional channels (i.e., mail and telephone), and the technology embracers, who like technologically advanced contacts (i.e., email). A simula- 


\section{FURTHER READING}

tion of varied resource allocations across the three channels for this group of customers produces the outcomes in Figure 2. That is, with equal telephone and mail contacts, but no email, positive reciprocity is likely among the first customer segment of traditionalists. However, it also may provoke negative reactance among the technology embracers. In contrast, dedicating all resources to email contacts initiates positive reciprocity among technology embracers and negative reactance among traditionalists.

\section{$>$ Develop protocols that limit total communication}

What about equal numbers of telephone, email, and mail contacts? The simulation suggests that this overwhelming approach would provoke reactance from everyone, both traditionalists and technology embracers. With three or fewer total contacts, most customers likely respond positively, but once the marketer goes beyond that point, repurchasing decreases. As these results show, this approach may allow marketers to successfully avoid inefficient allocations of marketing resources.

They can do so by developing protocols that limit the total communication through all channels and specifying effective channel combinations. Taken together, the evidence from this study suggests that multichannel communication must be carefully managed on multiple dimensions to avoid generating reactance and potentially driving customers away from, rather than closer to, the company.
Reinartz, Werner, Jaquelyn S. Thomas, and V. Kumar (2005), "Balancing Acquisition and Retention Resources to Maximize Customer Profitability", Journal of Marketing, 69 (January), 63 - 79.

Rust, Roland T. and Peter C. Verhoef (2005), "Optimizing the Marketing Intervention Mix in Intermediate-Term CRM." Marketing Science, 24 (3), $477-89$.

Seiders, Kathleen, Glenn B. Voss, Dhruv Grewal, and Andrea Godfrey (2005), "Do Satisfied Customers Buy More? Examining Moderating Influences in a Retailing Context", Journal of Marketing, 69 (October), $26-43$.

Voss, Glenn B., Andrea Godfrey, and Kathleen Seiders (2010), "How Complementary and Substitution Effects Alter the Customer SatisfactionRepurchase Link", Journal of Marketing, 74 (November), 111 - 127.

\section{KEYWORDS:}

Multichannel Communication, Communication Mix, Direct Marketing, Relationship Management, Customer Reactance, Customer Repurchase 